\title{
HEMIANOPSIA HOMÓNIMA SECUNDARIA A MALFORMACIONES ARTERIOVENOSAS CEREBRALES EN LA INFANCIA
}

\author{
HOMONYMOUS HEMIANOPIA DUE TO ARTERIOVENOUS \\ MALFORMATIONS OF THE BRAIN IN INFANCY
}

\author{
PUERTAS-BORDALLO D ${ }^{1}$, CONESA-HERNÁNDEZ E², RUIZ-FALCÓ-ROJAS-ML ${ }^{3}$, \\ OTERO-OVILLENA MJ ${ }^{2}$, LOZANO-VÁZQUEZ M ${ }^{4}$, RAMÍREZ-LILLO N²
}

\section{RESUMEN}

Introducción: Las malformaciones arteriovenosas (MAV) son patologías de baja prevalencia pero responsable con relativa frecuencia de patología visual en la población joven.

El estudio de la malformación vascular ha de realizarse mediante angiografías selectivas que marquen tanto las aferencias arteriales como los drenajes venosos, además, éstas, ofrecen información muy valiosa para orientar el tratamiento.

Caso clínico: Se presenta un caso de hemianopsia homónima izquierda instaurada en un varón de diez años de edad con malformaciones arteriovenosas cerebrales temporo-occipitales derechas.

Discusión: Las malformaciones arteriovenosas cerebrales son lesiones con una morbimortalidad elevada. Para el oftalmólogo, tienen especial importancia debido a la posibilidad de provocar, dependiendo de su localización, sintomatología visual variada.

Palabras clave: Hemianopsia homónima, malformación arteriovenosa cerebral, angiografía, aferencias arteriales, drenajes venosos.
Introduction: Arteriovenous malformations (AVM) of the brain have a low prevalence but are responsible for a significant component of visual pathology in the young population. Vascular studies using selective angiography are necessary to identify arterial connections and venous drainage, and provide useful information for planning treatment. Clinical case: A 10-year-old child with a temporooccipital vascular malformation resulting in a left homonymous hemianopia.

Discussion: Arteriovenous malformations of the brain are lesions with a high morbidity and mortality. For the ophthalmologist, they are of special importance due to the possibility of causing a variety of visual defects, depending on their location (Arch Soc Esp Oftalmol 2007; 82: 635-640).

Key words: Homonymous hemianopia, arteriovenous malformation of the brain, angiography, arterial connections, venous drainage.

\footnotetext{
Recibido: 22/6/06. Aceptado: 20/9/07.

Sección de Oftalmología/Estrabología. Hospital Infantil Universitario Niño Jesús. Madrid. España.

1 Doctor en Medicina.

2 Licenciado en Medicina. MIR de Oftalmología. Hospital Central de la Cruz Roja de Madrid. España.

3 Licenciado en Medicina. Sección de Neurología.

${ }^{4}$ Licenciado en Medicina. MIR de Oftalmología. Complejo Hospitalario Universitario de Santiago de Compostela.

Correspondencia:

Diego Puertas Bordallo

Sección de Oftalmología/Estrabología.

Hospital Infantil Universitario Niño Jesús.

Avenida Menéndez Pelayo, 65

28009 Madrid

España

E-mail: dpuertas.hnjs@salud.madrid.org
} 


\section{INTRODUCCIÓN}

Las malformaciones arteriovenosas pueden estar localizadas en cerebro, tronco encefálico y médula espinal. Están compuestas por ovillos vasculares anómalos que comunican los sistemas arterial y venoso sin interposición de lecho capilar alguno. Pueden ser de variado tamaño, siendo las más voluminosas, con mayor frecuencia, aquellas localizadas en la mitad posterior de los hemisferios (1).

Tienen una prevalencia de aproximadamente $0,01 \%$ a $0,5 \%$ de la población. Aunque son lesiones congénitas, las posibles complicaciones suelen ocurrir con mayor frecuencia entre los 10 y 40 años.

Los síntomas clínicos más importantes son la cefalea hemicraneal, las crisis epilépticas y los debidos a la hemorragia cerebral intraparenquimatosa, que aparece en el 50\%-70\% de los casos, siendo así la manifestación clínica más frecuente. De producir defectos neurológicos progresivos, son los defectos visuales hemianópsicos, los más frecuentes (2).

En el diagnóstico por imagen, la TC con contraste puede detectar MAV antes de su rotura. La RMN tiene aún mayor sensibilidad. Sin embargo, el estudio de la malformación vascular ha de realizarse mediante arteriografías selectivas que marquen tanto las aferencias arteriales como los drenajes venosos, además, éstas, ofrecen información muy valiosa para orientar el tratamiento.

El tratamiento es controvertido, pudiéndose adoptar una postura contemplativa, o bien, decantarse por otra más activa: la radiología intervencionista, la radioterapia estereotáctica o convencional y el abordaje quirúrgico suponen las técnicas habituales, no exentas, a su vez, de complicaciones generales y neurológicas sobreañadidas (3).

\section{CASO CLÍNICO}

Varón de diez años en la actualidad, que en 2001, sufrió, bruscamente, una cefalea intensa asociada a vómitos y refractaria a ibuprofeno. En la exploración de fondo de ojo, se advirtió un papiledema derecho incipiente. En la TC se objetivó una hemorragia occipital parasagital derecha (fig. 1).

Posteriormente se llevaron a cabo estudios angiográficos hallándose dos nidos malformativos, uno, en la cara medial del lóbulo temporal derecho, de 7,5 cc de volumen, y otro más posterior, localizado en el lóbulo occipital derecho, de $8 \mathrm{cc}$ de volumen,

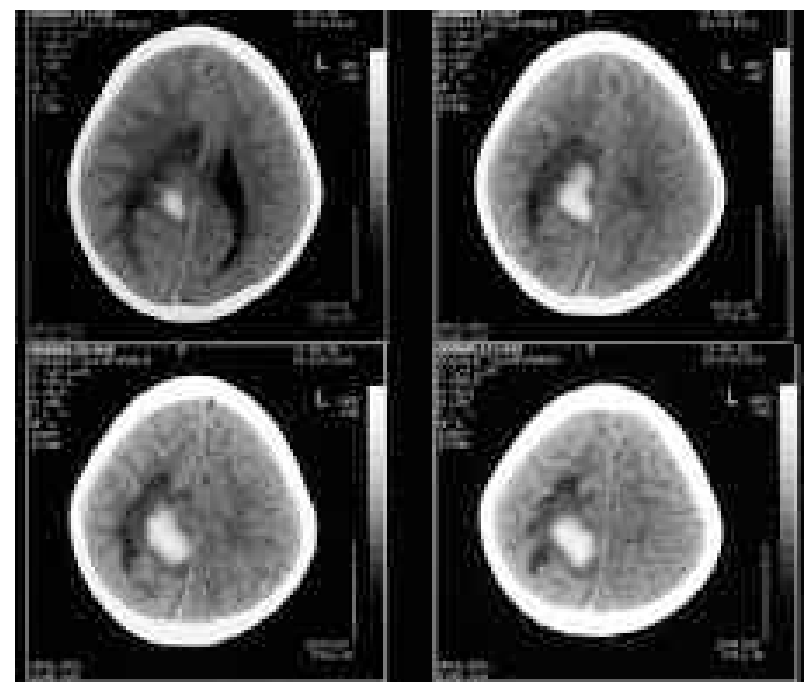

Fig. 1: TC cerebral. Se aprecian imágenes hipodensas compatibles con hemorragia parasagital derecha.

demostrándose asimismo angiogénesis periférica en ambos lóbulos La parte occipital de la MAV presentaba un mayor flujo que la porción anterior.

En la exploración neurológica se descubrió, por confrontación, una hemianopsia homónima izquierda sin otros hallazgos. El examen de fondo de ojo, ya alejados del episodio agudo de sangrado, era normal en ambos ojos.

Entre 2001 y 2005, el niño fue intervenido con embolizaciones selectivas de sus dos componentes malformativos, disminuyendo el flujo sanguíneo de las aferencias de las MAV y el tamaño de éstas.

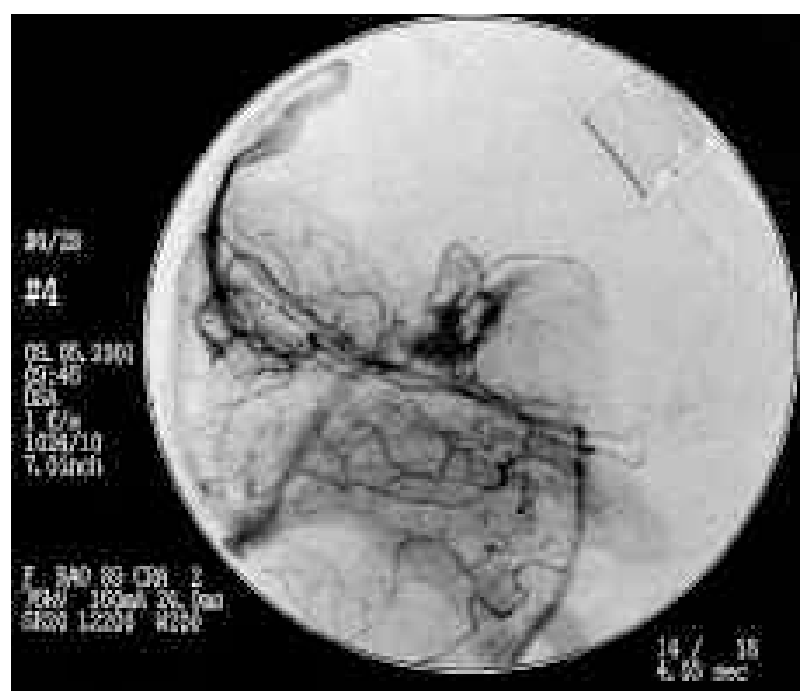

Fig. 2: Arteriografía en la que se hace visible un nido vascular malformativo occipital. 
Posteriormente se realizó radiocirugía con Gamma Knife (figs. 3a y 3b).

El paciente no ha sufrido recurrencia de la hemorragia pero presenta crisis epilépticas sintomáticas fotosensoriales en tratamiento médico con ácido valproico.

Su estudio electroencefalográfico muestra asimetría interhemisférica a expensas del hemisferio cerebral derecho, compatible con afectación cerebral estructural parieto-occipital derecha donde se han observado esporádicas anomalías epileptiformes localizadas en estas regiones.

En la actualidad, se ha incrementado la dosis farmacológica de su tratamiento médico por reagudización de las crisis y acude a nuestro servicio, remitido por el servicio de neurología, para valoración de diplopía intermitente, de cuatro meses de evolución, que en un principio se atribuyó a sus crisis epilépticas pero que no ha remitido pese al incremento farmacológico de ácido valproico.

En la exploración oftalmológica, el paciente tiene $\mathrm{AV}$ de $0,6(\mathrm{OD})$ y $0,5(\mathrm{OI})$. Los reflejos pupilares son normales. Presenta ortoforia, con ducciones y versiones normales. En la prueba del Cover Test, se demuestra una exoforia de $-3^{\circ}-5^{\circ}$ de cerca y $-5^{\circ}$

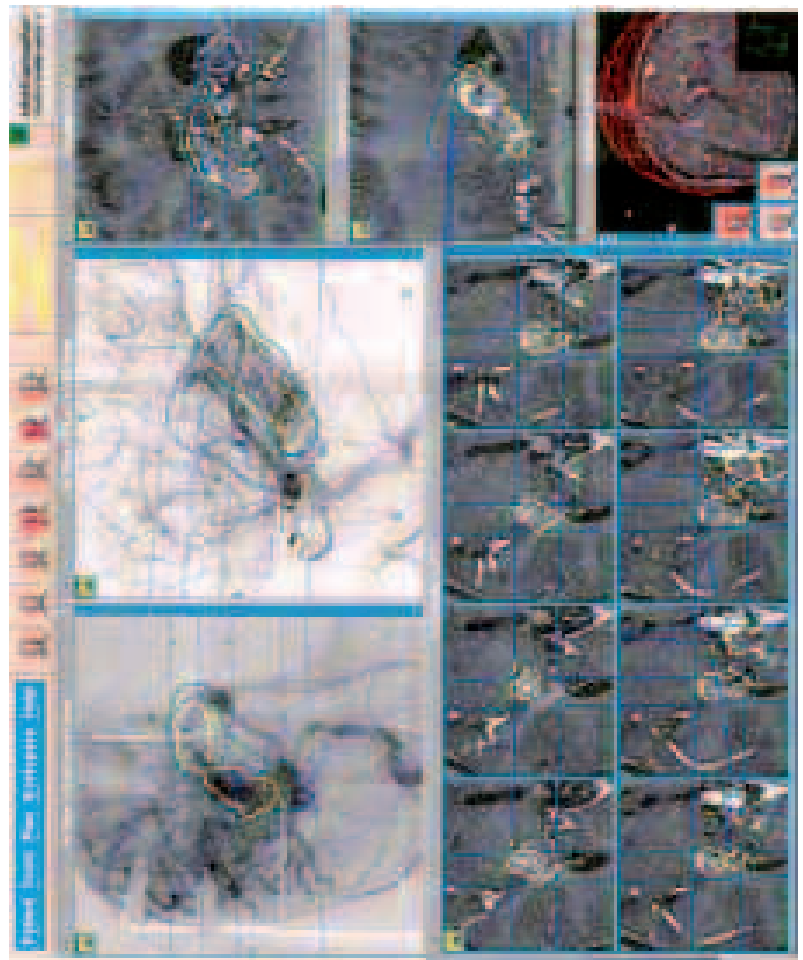

$-7^{\circ}$ de lejos. El fondo de ojo de ambos ojos es aparentemente normal. El defecto refractivo evidencia miopía de $-0,75$ dioptrías en ambos ojos. Se lleva a cabo estudio campimétrico (Humphrey, prueba de umbral 24-2) demostrándose defecto hemianópsico homónimo izquierdo altamente congruente en ambos ojos, y con mayor densidad del defecto en el cuadrante inferior izquierdo y respeto macular (figs. 4a y $4 \mathrm{~b}$ ). Se prescribe corrección óptica al paciente con la finalidad de mejorar su agudeza visual y controlar su foria.

\section{DISCUSIÓN}

Las MAV cerebrales son lesiones capaces de generar clínica oftalmológica por afectación de la vía visual. La vía óptica retroquiasmática está constituida por fibras que llevan información de los hemicampos contralaterales. Por esta razón, sus lesiones producen hemianopsias homónimas contralaterales, con característica particulares según la localización del daño que se produzca. A medida que éste se sitúe más posterior y cercano a la corteza occipital, el defecto campimétrico se va volvien-

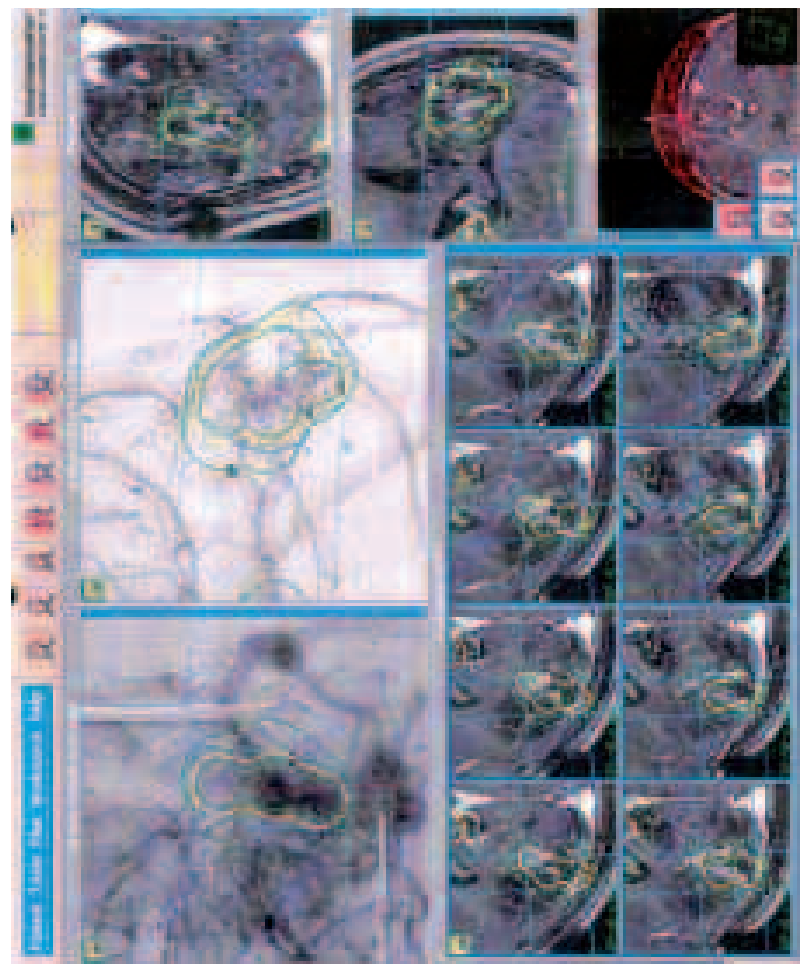

Fig. 3: Angio-resonancias seriadas con cortes en diferentes planos para orientar la Gamma Knife. 

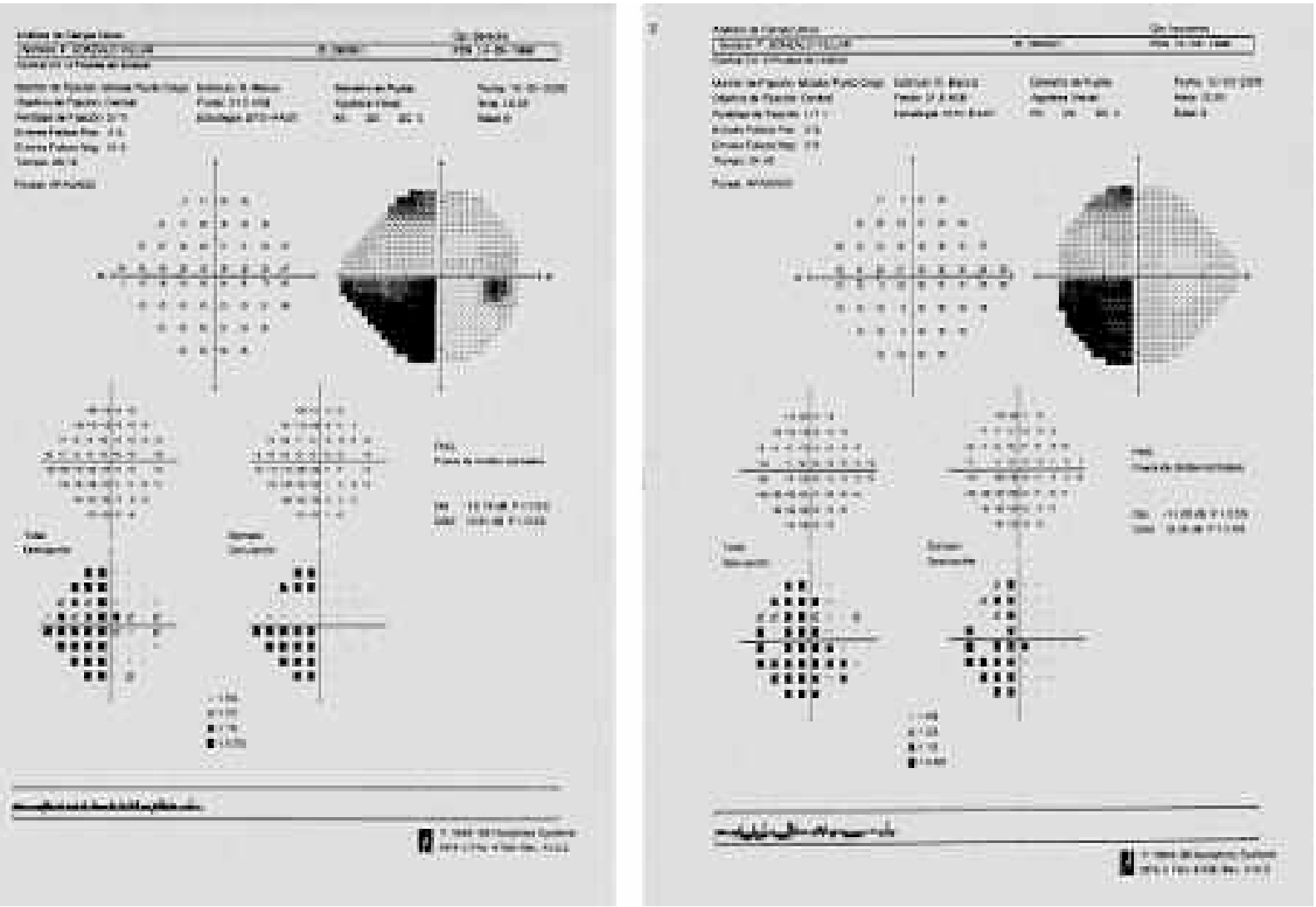

Fig. 4: Defectos campimétricos de OD y OI mostrando hemianopsia homónima izquierda de gran congruencia.

do cada vez más congruente, esto es, los defectos de los campos visuales de ambos ojos se van asemejando, con un grado máximo de similitud cuando la lesión de la vía óptica se sitúa en el lóbulo occipital. Asimismo, en este territorio cerebral, puede haber respeto macular, debido a la doble irrigación del área de representación macular. Las MAV suponen un pequeño porcentaje de las lesiones occipitales, pero con independencia de la causa, las alteraciones del campo visual y las crisis epilépticas son manifestaciones clínicas características a todas ellas (2).

No se conoce con exactitud la asociación establecida entre las MAV occipitales y la producción de defectos visuales. No hay una demostración de que exista correlación entre el tamaño de la malformación y el grado de defecto campimétrico desarrollado (3).

Al ser las MAV cerebrales lesiones congénitas, y de crecimiento lento, es frecuente que los daños que producen queden asintomáticos, y sólo sean diagnosticados si los defectos son obvios o si se realizan pruebas diagnósticas por otra causa. Entre los mecanismos patogénicos propuestos, de los defec- tos campimétricos, se contemplan compresiones directas de la vía visual, infartos, hemorragias y fenómenos de robo sanguíneo que conllevan isquemias parciales por hipoperfusión tisular.

Aunque se ha descrito algún caso de MAV occipital con degeneración neuronal retrógrada secundaria y atrofia sectorial papilar con desarrollo de defecto pupilar, ésto no es lo habitual (4).

Por último, recordamos que la resección quirúrgica o bien la radiocirugía estereotáctica, con embolización previa o no, de estas lesiones, pueden llevarse a cabo con bajo riesgo de causar nuevos defectos visuales o de empeorar los ya establecidos, e incluso algunos autores advierten que intervenciones precoces pueden mejorar o incluso resolver muchos casos de defectos visuales secundarios a estas lesiones vasculares cerebrales (5).

\section{BIBLIOGRAFÍA}

1. Easton JD, Hauser SL, Martin, JB. Enfermedades cerebrovasculares. In: Fauci AS. Harrison. Principios de 
Medicina Interna. Madrid. McGraw-Hill-Interamericana de España; 1998; II: 2671-2672.

2. Gutteridge IF, McDonald RA. Hemianopic visual field loss as the first clinical evidence of occipital arteriovenous malformation. Clin Exp Optom 2004;87: 394399.

3. Bartolomei J, Wecht DA, Chaloupka J, Fayad P, Awad IA. Occipital lobe vascular malformations: prevalence of visual field deficits and prognosis after therapic intervention. Neurosurgery 1998; 43: 415-421.

4. Rodallec T, Fiandrino P, Iba-Zizen MT, Cabanis E, Gastaud P, Normand JP et al. Arteriovenous malformation of the brain revealed through a visual blur and Adie's pupil. J Fr Ophtalmol 2005; 28: 401-406.

5. Hillman J. Population-based analysis of arteriovenous malformation treatment. J Neurosurg 2001; 95: 633-637. 